\title{
Knockdown of metadherin inhibits angiogenesis in breast cancer
}

\author{
YAN LIU ${ }^{1,2}$, XIANGNAN KONG ${ }^{1}$, XIAOYAN LI ${ }^{1},{\text { BAOJIANG } \text { LI }^{2} \text { and QIFENG YANG }}^{1,3}$ \\ ${ }^{1}$ Department of Breast Surgery, Qilu Hospital, Shandong University, Jinan, Shandong 250012; \\ ${ }^{2}$ Department of Breast Surgery, Taian City Central Hospital, Taian, Shandong 271000; \\ ${ }^{3}$ Department of Pathology Tissue Bank, Qilu Hospital, Shandong University, \\ Jinan, Shandong 250021, P.R. China
}

Received February 20, 2015; Accepted April 2, 2015

DOI: 10.3892/ijo.2015.2973

\begin{abstract}
Angiogenesis plays an important role in cancer growth, invasion and metastasis. It has been confirmed that metadherin (MTDH) is associated with angiogenesis. However, the detailed mechanism of MTDH on angiogenesis has not yet been reported. In this study, we demonstrate the anti-angiogenic function of MTDH in breast cancer. With RNA interference strategies, we found that knockdown of MTDH inhibits cellular angiogenesis both in vitro and ex vivo. Furthermore, we revealed that ERK1/2 pathway is involved in the anti-angiogenic function of $\mathrm{MTDH}$, and the function can be partially reversed via upregulation of microRNA-21 (miR-21). In conclusion, knockdown of MTDH can inhibit angiogenesis in breast cancer. These results show that MTDH is a viable therapeutic target for anti-angiogenesis in breast cancer.
\end{abstract}

\section{Introduction}

Breast cancer is the most frequently diagnosed cancer and leading cause of cancer deaths among women in the world. Currently, breast cancer is the most common cancer among women in China; new cases account for $12.2 \%$ and the mortality rate is $9.6 \%$ of all breast cancer patients worldwide (1). In 2013, a total of 232,340 cases of invasive breast cancer and 39,620 breast cancer deaths were reported among US women (2). Although the mortality has dropped over the past decades, distant metastasis is still a main cause of death among breast cancer.

Correspondence to: Professor Qifeng Yang, Department of Breast Surgery, Qilu Hospital, Shandong University School of Medicine, 44 Wenhua Xi Road, Jinan, Shandong 250012, P.R. China

E-mail: qifengy@gmail.com

Professor Baojiang Li, Department of Breast Surgery, Taian City Central Hospital, 29 Longtan Road, Taian, Shandong 271000, P.R. China

E-mail: bjiangli@hotmail.com

Key words: metadherin, angiogenesis, anti-angiogenesis, miR-21, breast cancer
By supplying nutrients and providing the vascular route for haematogenous metastasis, vascular-dependent diseases such as breast cancer can be affected by angiogenesis (3-5). Since angiogenesis plays a pivotal role in breast cancer development, and seriously effects cancer cell invasion and metastasis, inhibition of tumor angiogenesis is considered as an attractive and effective strategy for the therapy of breast cancer (6). Angiogenesis is a complex process, which is regulated by different molecular pathways (7).

Metadherin (MTDH), as a novel multifunctional oncogene, originally identified in 2002 (8). In our previous studies, we have found that MTDH improves the invasiveness of breast cancer cells by inducing epithelial to mesenchymal transition, is involved in inflammation-induced tumor progression, modulates TRAIL-resistance in breast cancer cells through caspase- 8 downregulation and Bcl-2 upregulation, mediates estrogen-independent growth and tamoxifen resistance through PTEN downregulation and that $>40 \%$ tumors overexpress MTDH, which correlates with metastasis and poor-prognosis of breast cancer (9-13). MTDH is frequently overexpressed in tumor tissues and its expression level is associated with the progression and worse prognosis of malignant tumor such as hepatocellular carcinoma, lung cancer, bladder cancer, laryngeal squamous cell carcinoma and breast cancer (14-19) Therefore, knockdown of MTDH can significantly inhibit prostate cancer progression, sensitize endometrial cancer cells to cell death induction by TRAIL and sensitize breast cancer cells to AZD6244 (20-22). In this study, we explored the inhibition of angiogenesis through knockdown of MTDH in breast cancer and the potential of MTDH as a therapeutic target for anti-angiogenesis.

\section{Materials and methods}

Reagents. Antibodies against ERK1/2, p-ERK, PTEN, MMP-2 and VEGF were purchased from Cell Signaling Technology (Beverly, MA, USA). Anti-MTDH rabbit antibody was obtained from Invitrogen (Carlsbad, CA, USA). Anti-CD31 antibody was obtained from Santa Cruz Biotechnology (Santa Cruz, CA, USA). The other reagents were obtained from Sigma-Aldrich (St. Louis, MO, USA).

Cell culture. Breast cancer cell line MDA-MB-231 and human umbilical vein endothelial cells (HUVECs) were purchased 
from American Type Culture Collection. They were cultured in Dulbecco's modified Eagle's medium supplemented with $10 \%$ fetal bovine serum (FBS) at $37^{\circ} \mathrm{C}$ and under $5 \% \mathrm{CO}_{2}$ incubator.

Plasmidconstructionandtransfection. The MTDHknockdown plasmids were constructed as previously described (23). In brief, the 19-nt sequence 5'-ATGAACCAGAATCAGTCAGC-3' was used to construct MTDH shRNA. The 60-nt oligonucleotides were annealed and inserted into the pSUPER.retro.pure (OligoEngine, Seattle, WA, USA). According to the manufacturer's protocol, the MDA-MB-231 cells were transfected with Lipofectamine 2000 (Invitrogen). The shRNA interference vector was applied to establish the MDA-MB-231-prpM cell line and the empty vector was used to establish the MDA-MB-231-prpn cell line. Cells were selected with $0.5 \mu \mathrm{g} / \mathrm{ml}$ puromycin to generate stable cell lines. miR-21mimics and the corresponding negative control (NC) (Gene Pharma, Shanghai, China) were used for upregulation of miR-21. Transiently transfected cells were harvested at $48 \mathrm{~h}$ for mRNA and at $72 \mathrm{~h}$ for protein analysis.

Quantitative real-time PCR. Total RNA was isolated using TRIzol by the manufacturer's protocol (Takara, Dalian, China). Total RNA was reverse transcribed to cDNA by applying Prime Script RT reagent kit (Takara). Quantitative RT-PCR was performed with the SYBR green detection (Takara) in Applied Biosystems StepOne Plus Real-Time PCR System (Applied Biosystems, Carlsbad, CA, USA). The level of GAPDH was used as the endogenous control for detection of mRNA expression analysis and U6 was used for microRNAs. The $2^{-\Delta \Delta C t}$ method was employed for data analysis.

Western blot analysis. Cells were harvested and lysed in ice-cold RIPA buffer (1X PBS, 1\% NP40, 0.1\% SDS, $5 \mathrm{mM}$ EDTA, $0.5 \%$ sodium deoxycholate and $1 \mathrm{mM}$ sodium orthovanadate) with protease and phosphatase inhibitors. The proteins were quantified using the BCA Protein Assay kit (Merck, Darmstadt, Germany). Same amount of protein was separated by $10 \%$ SDS-PAGE gel and then transferred onto PVDF membrane (Millipore, Bedford, MA, USA). After blocking with $5 \%$ non-fat dry milk, the PVDF membrane was first incubated overnight at $4^{\circ} \mathrm{C}$ with primary antibodies, rinsed and incubated with the secondary antibodies. The corresponding signals were detected with enhanced chemiluminescence (ECL).

Tube formation assay. Tumor cell conditioned medium (TCM) without FBS was obtained as described (24). The 96-well plates were covered with $50 \mu \mathrm{l}$ Matrigel and placed at $37^{\circ} \mathrm{C}$ for $30 \mathrm{~min}$ to polymerize. The HUVECs were suspended using different TCM and $100 \mu \mathrm{l}$ of HUVECs were added to each well. Then HUVECs were incubated for $7 \mathrm{~h}$. Tube-like structures were photographed with an Olympus digital camera and macroscopic quantities were recorded by counting at least 10 microscopic fields.

Mouse aortic ring assay. Mouse aortas were dissected from $\mathrm{BALB} / \mathrm{c}$ mice and cut into $\sim 1$-mm long sections as previously described (25). A 48-well plate was first covered with $100 \mu 1$ of Matrigel and incubated for $30 \mathrm{~min}$ at $37^{\circ} \mathrm{C}$. The aortic rings were put into the wells and then covered with an overlay of $100 \mu \mathrm{l}$ Matrigel, followed by addition of $200 \mu \mathrm{l}$ of TCM. The cultures were kept at $37^{\circ} \mathrm{C}$ in a humidified environment for a week and the result of the fields covered by sprouting from the aortic rings was examined with an Olympus microscope at appropriate magnification.

Immunohistochemistry (IHC). Seventy-seven breast cancer tissue samples were obtained from the Department of Pathology of Qilu Hospital of Shandong University from 2011 to 2014. To quantify the microvessel density (MVD), the SP-9000 Histostain ${ }^{\mathrm{TM}}$-Plus kits (Zhongshan Goldenbridge Biotechnology Co.) were used to detect CD31 expression following standard steps as previously described $(26,27)$.

MicroRNA array analysis. Total RNA was isolated using TRIzol by the manufacturer's protocol. A microarray with 873 miRNA probes was designed in accordance with Sanger miRbase release 12.0. RNA labeling and hybridization were performed as previously described (28). After hybridization, microarrays were investigated by the LuxScan 10K Microarray Scanner (CapitalBio, Beijing, China), and the images were analyzed by GenePix Pro 6.0 software (Axon Instruments, Foster City, CA, USA). The data are available in the Gene Expression Omnibus (GEO).

Statistical analysis. Statistical software SPSS 18.0 was used. The data are shown as mean \pm SD. The difference in statistics was analyzed through the Student's t-test and regarded as statistically significant for P-values $<0.05$.

\section{Results}

Establishment of the MTDH knockdown cell line. Due to the high level expression of MTDH in MDA-MB-231 cells, we first designed the short hairpin RNA and then transfected the plasmid into MDA-MB-231 cells to establish the MTDH knockdown cell line. The stable cell line was selected by adding puromycin to DMEM. MTDH expression levels were detected by qRT-PCR and western blot analysis. As shown in Fig. 1, MTDH expression levels were obviously lower in MDA-MB-231-prpM cells than that in control MDA-MB-231-prpn cells.

Knockdown of MTDH inhibits angiogenesis in vitro. To confirm whether the knockdown of MTDH could inhibit angiogenesis of breast cancer cells, a tube formation assay was performed as an important indicator of endothelial function in angiogenesis. HUVECs cultured on Matrigel rapidly align and finally form tube-like structures. Since the tube formation can be affected by different medium components, TCM obtained from different breast cells was added to HUVECs cultured on Matrigel and the tube-like structures were quantitatively investigated. Compared to the control MDA-MB-231-prpn cells, HUVECs cultured with TCM from MDA-MB-231-prpM cells caused an obvious decrease in tube formation, as shown in Fig. 2A.

Knockdown of MTDH inhibits angiogenesis ex vivo. To investigate the inhibition of angiogenesis ex vivo, we detected 
A
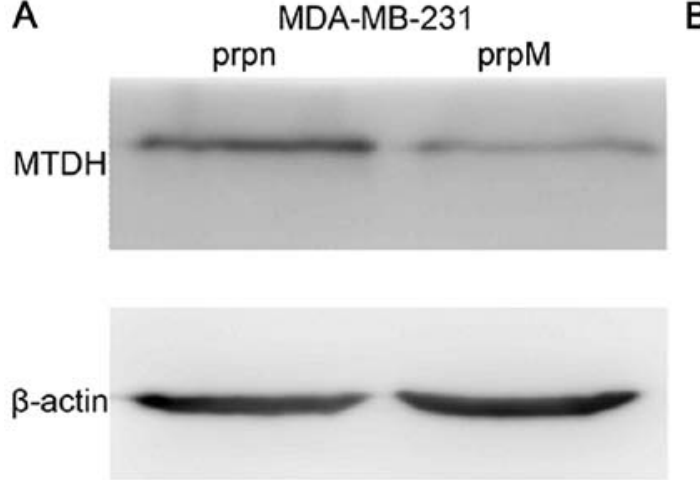

B

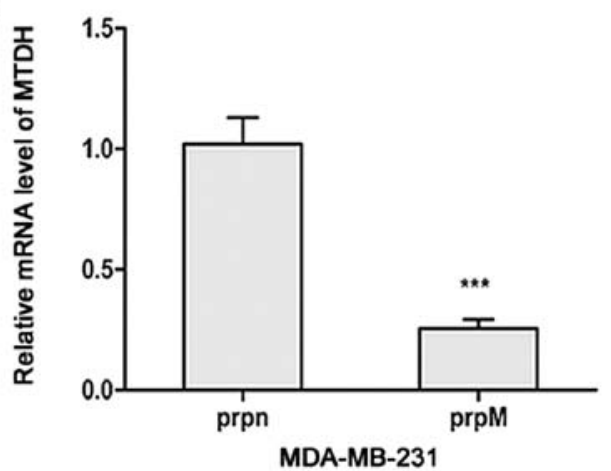

Figure 1. Knockdown of MTDH in the MDA-MB-231 cell line. (A) Protein levels of MTDH were validated through western blot analysis. (B) The mRNA levels of MTDH were validated by qRT-PCR. The panels show the data from three independent experiments. ${ }^{* * *} \mathrm{P}<0.01$.

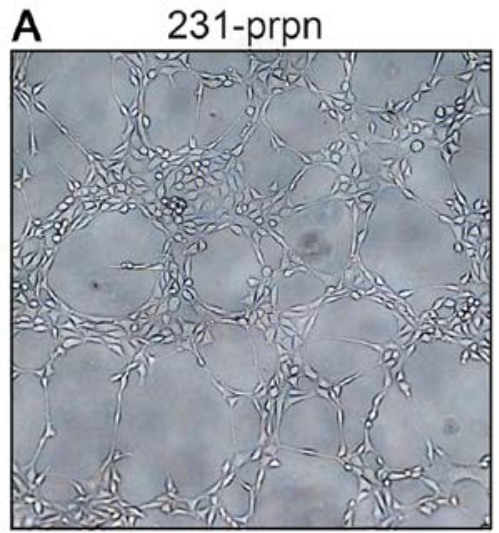

B

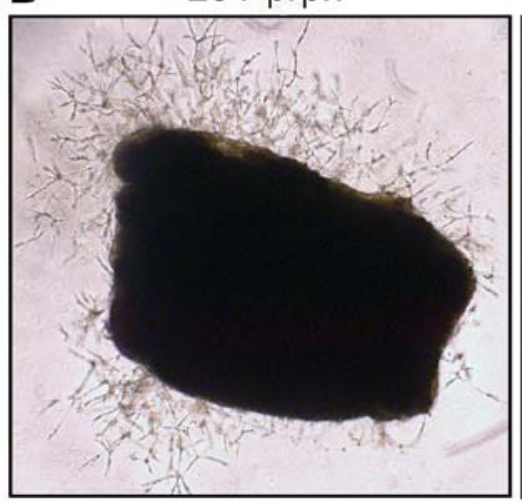

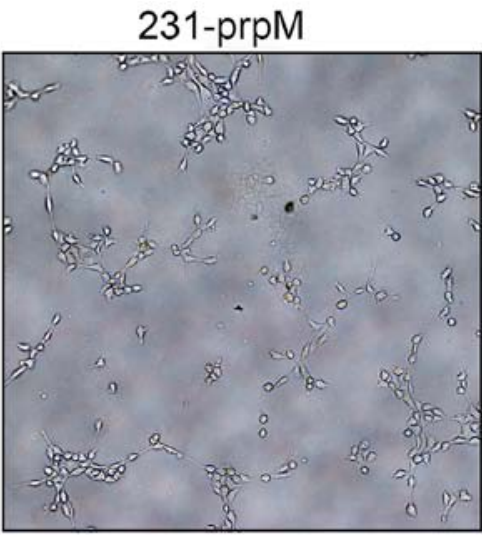

231-prpM

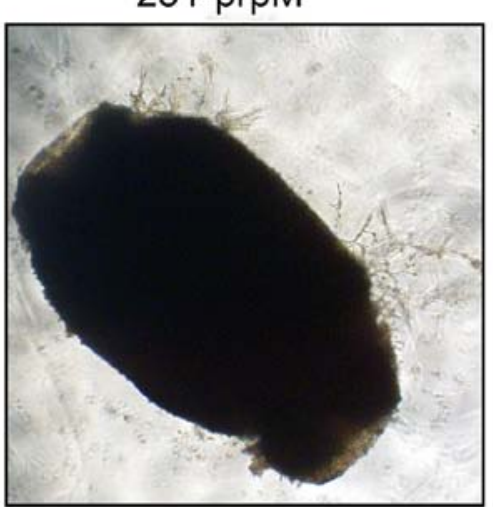

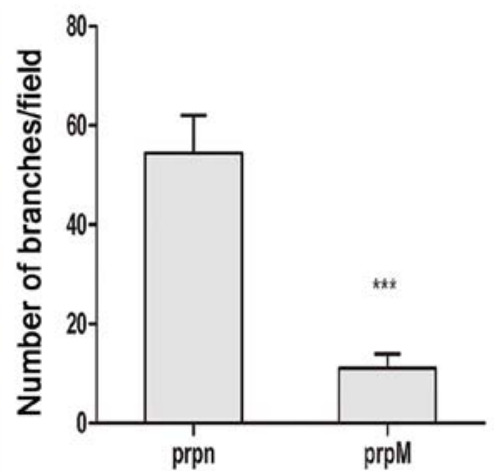

MDA-MB-231

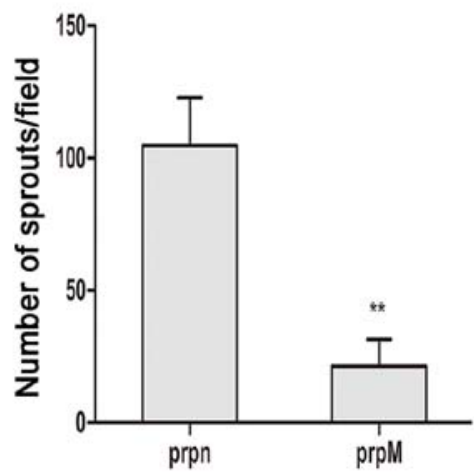

MDA-MB-231

Figure 2. Knockdown of MTDH inhibited angiogenesis. (A) HUVECs were suspended using different TCM and incubated on Matrigel-covered 96-well plates. Tube-like structures were assessed after $7 \mathrm{~h}$. Original magnification, $\mathrm{x} 100$. The bar graph shows the number of tube-like structures. (B) Mouse aortic rings were cultured in different TCM. The images of sprouting at day 7. Original magnification, x100. The bar graph shows the number of sprouts. Data are from three independent experiments. ${ }^{* *} \mathrm{P}<0.05 .{ }^{* * *} \mathrm{P}<0.01$.

the sprouting of vessels from mouse aortic rings. Mouse aortas cultured in Matrigel were treated with TCM from MDA-MB-231-prpM cells and the control group. The effect on angiogenesis of MTDH was demonstrated through comparing the fields covered by sprouting from the aortic rings. As shown in Fig. 2B, the knockdown of MTDH significantly inhibited the formation of microvessel structures around the mouse aortic rings.
The knockdown of MTDH downregulates the expression of p-ERK1/2 and decreases the levels of VEGF and MMP2. ERK1/2 molecule was widely regarded as a signal pathway activator of angiogenesis $(29,30)$. Therefore, the protein levels of ERK $1 / 2$ and p-ERK $1 / 2$ were monitored in our test to explore a potential mechanism of action. As shown in Fig. 3, the knockdown of MTDH downregulated the expression of p-ERK1/2 in western blot analysis. Because p-ERK1/2 could 
A

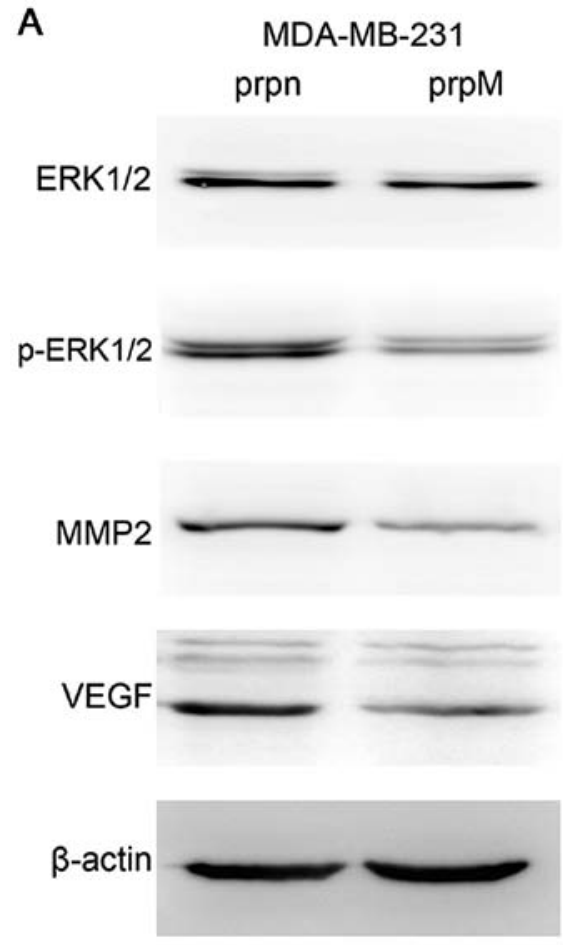

B
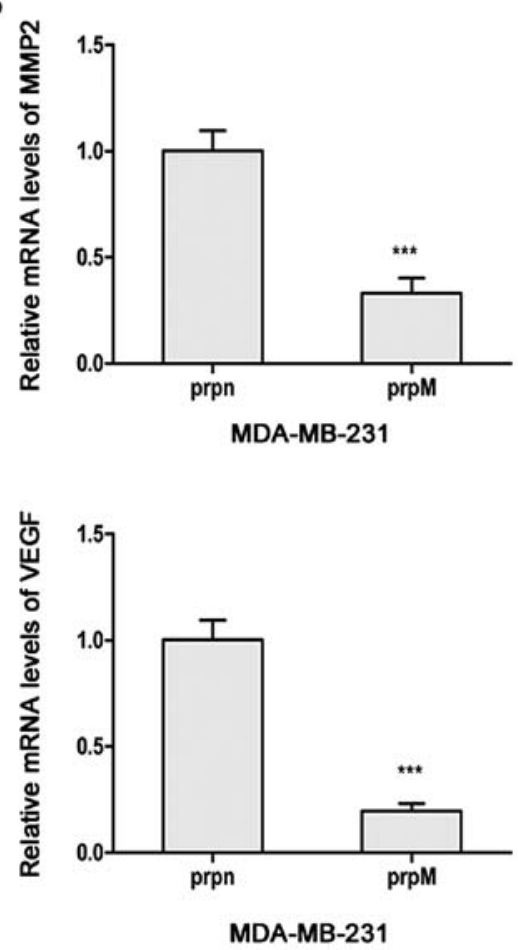

Figure 3. Knockdown of MTDH inhibits the ERK1/2 pathway in MDA-MB-231 cells. (A) The protein levels of ERK1/2 p-ERK1/2 MMP-2 and VEGF was analyzed using western blot analysis. $\beta$-actin was employed as the loading control. (B) The mRNA levels of MMP-2 and VEGF were assessed by qRT-PCR. ${ }^{* * * * *} \mathrm{P}<0.01$.

A

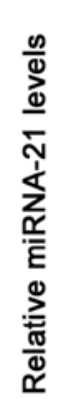

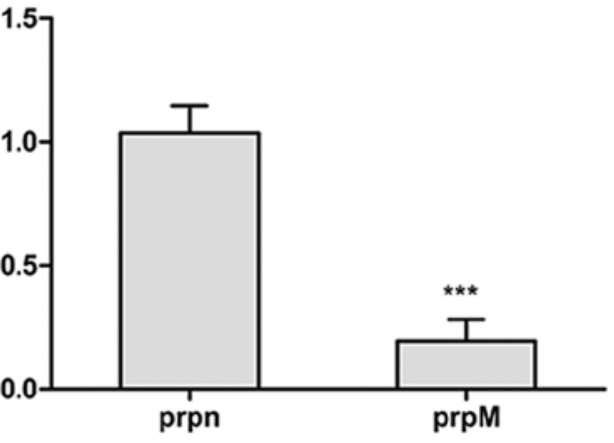

MDA-MB-231
B

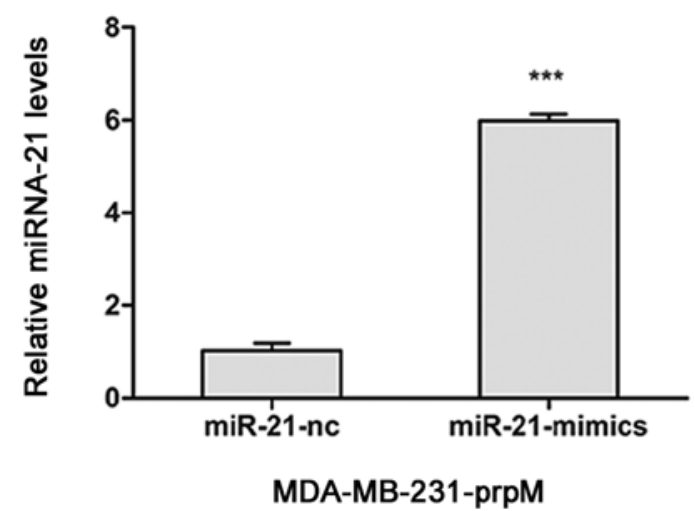

Figure 4. miR-21 levels were assessed using qRT-PCR. (A) The miR-21 levels were detected in MDA-MB-231-prpM cells and control. (B) The miR-21 levels were detected after transfection of miR-21-mimics in MDA-MB-231-prpM cells.

regulate angiogenesis through MMP-2 and VEGF (31), we measured the mRNA levels and protein levels of MMP-2 and VEGF in MTDH knockdown cells. The levels of the two markers were significantly reduced in MDA-MB-231-prpM cells.

miR-21-mimics increase miR-21-inducing ERK1/2, MMP2 and VEGF expression, and promote tumor angiogenesis. To investigate the influence of MTDH knockdown on the inhibition of angiogenesis, by regulating levels of miRNAs, the miRNA arrays was adopted to detect the changes of miRNAs after MTDH knockdown. From the miRNA array data, we found MTDH regulated miRNA expression in MDA-MB-231-
prpM cell (data not shown). Among them, miR-21 level was significantly decreased. To investigate whether miR-21 was involved in the inhibition of angiogenesis, we transected miR21-mimics with Lipofectamine 2000 into MDA-MB-231-prpM to upregulate the level of miR-21. As shown in Fig. 4, the level of miR-21 obviously increased. Then, we applied tube formation assay and mouse aortic ring assay to explore the role of miR-21 in angiogenesis. The results showed that upregulated expression of miR-21 could partially reverse the inhibition of angiogenesis in MDA-MB-231-prpM cells (Fig. 5). Previous studies indicated that miR-21 regulated expression of PTEN $(32,33)$. Therefore, we detected PTEN and p-PTEN in miR-21 mimic-transfected MDA-MB-231-prpM cells. As shown in 
A
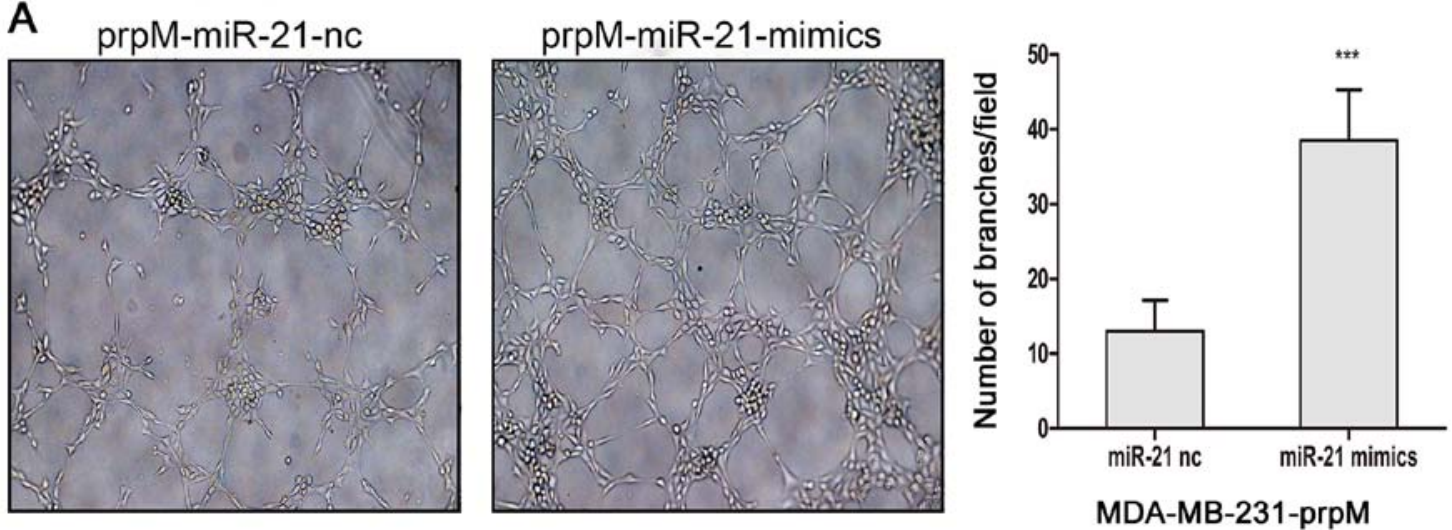

B
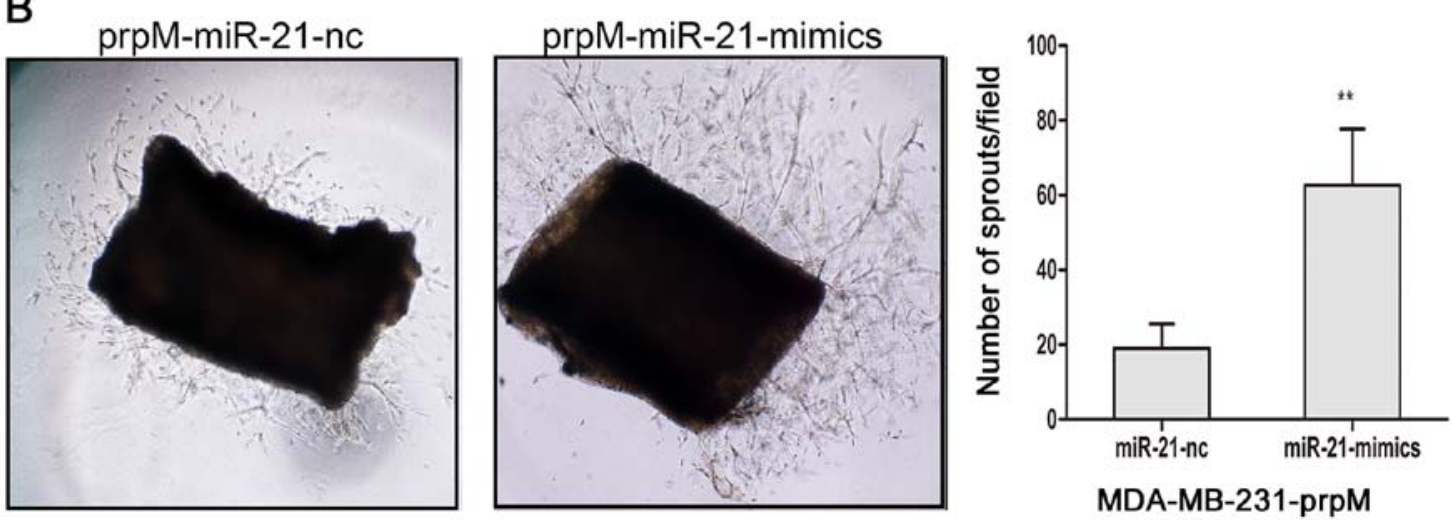

Figure 5. Transfection of miR-21-mimics partially reversed the inhibition of angiogenesis in MAD-MB-231-prpM cells. (A) Effect of the miR-21-mimics on the tube formation of HUVECs. Tube-like structures were assessed after $7 \mathrm{~h}$. Original magnification, x100. The bar graph shows the number of tube-like structures. (B) Effect of the miR-21-mimics on the aortic ring assay. The bar graph shows the number of sprouts. Original magnification, $\mathrm{x} 100$. Data are from three independent experiments. ${ }^{* *} \mathrm{P}<0.05$. $^{* * *} \mathrm{P}<0.01$.

A

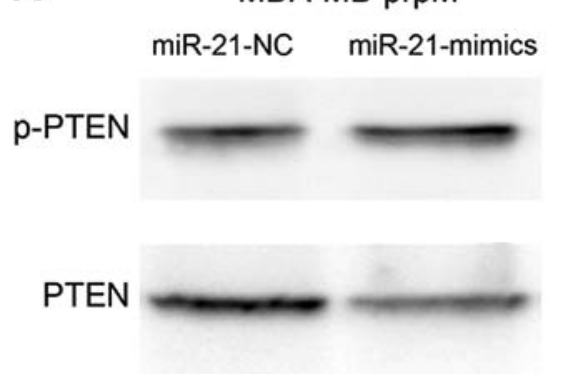

p-ERK1/2

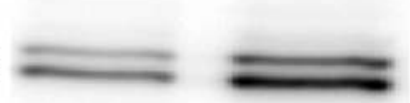

MMP2

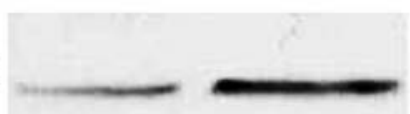

VEGF

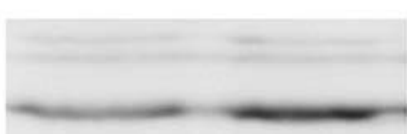

$\beta$-actin
B
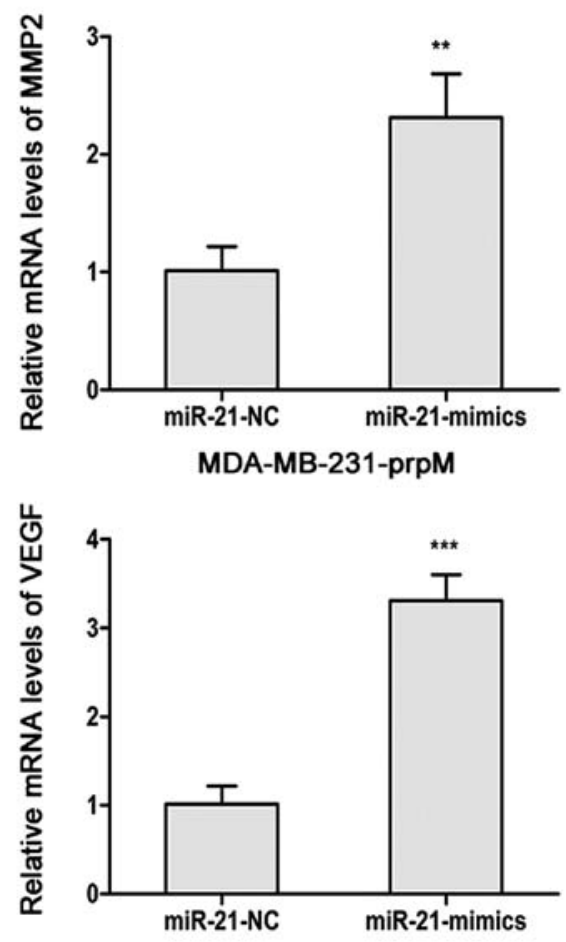

MDA-MB-231-prpM

Figure 6. The transfection of miR-21-mimics promotes the ERK1/2 pathway in MDA-MB-231-prpM cells. (A) The protein levels of PTEN ERK1/2 p-ERK1/2 MMP-2 and VEGF were analyzed using western blot analysis. (B) The mRNA levels of MMP-2 and VEGF were measured using qRT-PCR. ${ }^{* * *} \mathrm{P}<0.01$. 

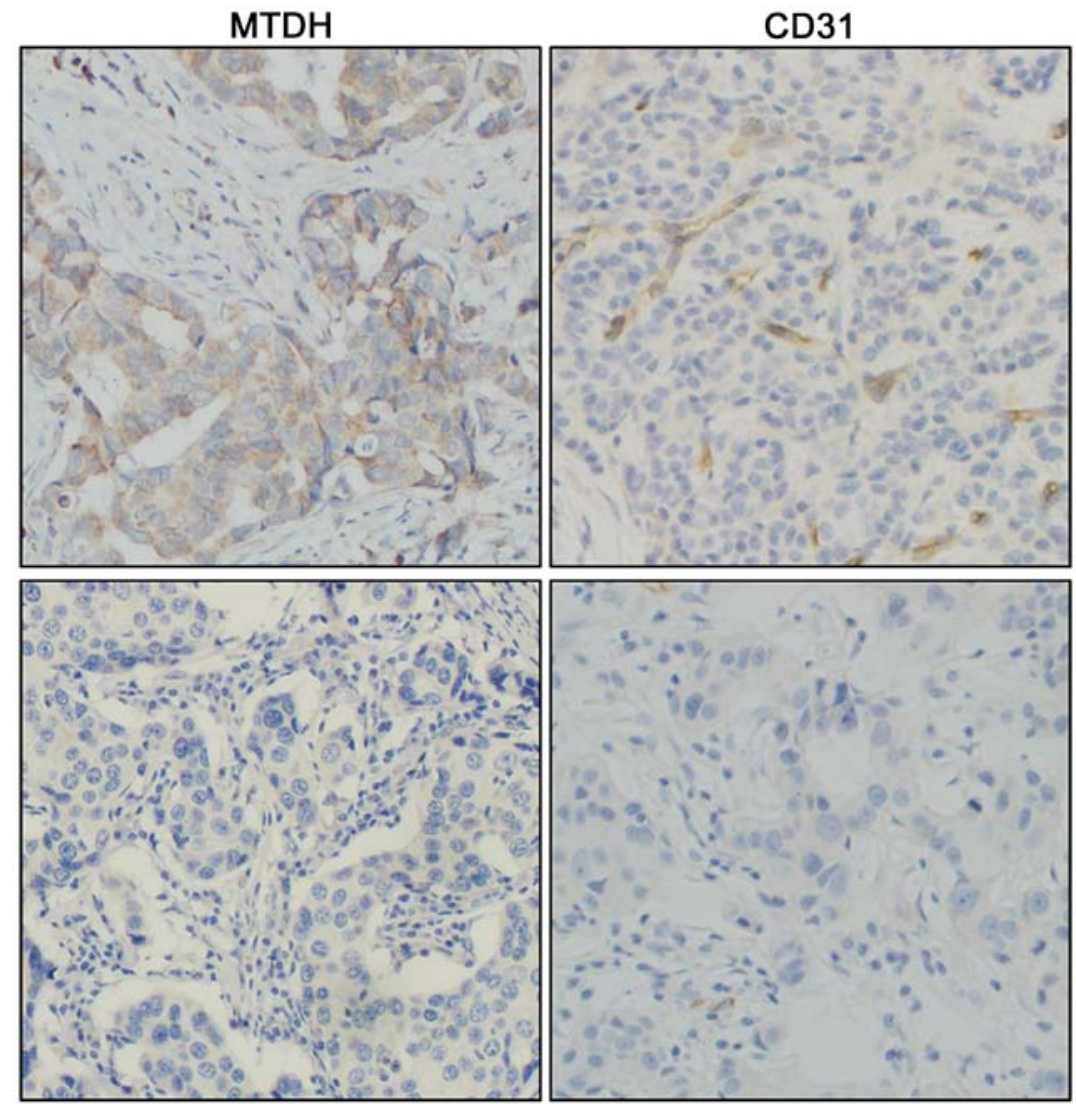

Figure 7. Sections of breast cancer samples were stained for MTDH and CD31. Representative images of sections with low expression of MTDH and CD31 (bottom), the sections with high expression of MTDH and CD31 (top).

Fig. 6, upregulated expression of miR-21 increased the protein level of p-ERK1/2 via suppressing PTEN, and then increased the levels of MMP2 and VEGF.

Lower MVD is linked with low expression of MTDH. To confirm the relationship between MTDH and angiogenesis in breast cancer tissue, we stained 77 breast cancer tissue samples with the MTDH and CD31 antibody. As shown in Fig. 7, 43 cases were CD31-positive in the $61 \mathrm{MTDH}$-positive cases in total. Of these, 6 cases were CD31-positive in the total 16 MTDH-negative staining cases. Therefore, compared to the MTDH overexpressing tissues, the result showed that low expression of MTDH was linked with lower MVD. The data were evaluated with the $\chi^{2}$ test $(\mathrm{P}=0.032)$.

\section{Discussion}

Angiogenesis is mediated by multiple molecules including vascular endothelial growth factor (VEGF), epiderma1 growth factor (EGF), epidermal growth factor receptor (EGFR) and matrix metalloproteinases (MMPs).

Due to the increased secretion of pro-angiogenic factors, malignant cells become more angiogenic originating from the activation of the oncogene or inactivation of the tumor suppressor gene. For example, in breast cancer, P53 mutations promote angiogenesis by upregulation of EGFR (34); activated RhoA promotes VEGF expression and angiogenesis by decrease of P53 stability (35); 53BP1 inhibits angiogenesis by decrease of MMP2 and MMP9 (36). Previous studies demonstrated that MTDH as an oncogene promotes invasion and metastasis of malignant cells. In triple-negative breast cancer, it was reported that MTDH correlates with angiogenesis and worse clinical outcomes through immunohistochemical staining of 125 specimens. However, the mechanism was not clarified (37).

To explore the function of the knockdown of MTDH to modulate angiogenesis in breast cancer, the expression of MTDH was manipulated with RNA interference in MDA-MB-231 cells. Our results showed that the knockdown of MTDH was able to suppress tube formation of HUVECs and sprouting of the mouse aortic rings. To further investigate the potential molecular mechanism of the knockdown of MTDH in inhibition of angiogenesis, we focused on the ERK1/2 signaling pathway, which is essential in cell proliferation, differentiation, apoptosis and angiogenesis $(29,38,39)$. The knockdown of MTDH downregulated the level of p-ERK1/2 in MDA-MB-231-prpM cells, and then decreased the levels of MMP2 and VEGF. These results demonstrated that the knockdown of MTDH is an effective method of anti-angiogenesis in breast cancer and p-ERK1/2 signaling is an essential event in this process.

MicroRNAs (miRNAs), 20-25 nucleotides non-proteincoding RNAs, have been proven to be involve in regulation of gene expression (40). The function of miRNAs have been confirmed not only in many biological processes but also in various pathological situations including cancer (41). 
Angiogenesis is a key process in cancer development, and the process can also be regulated by many miRNAs such as miR-29b in hepatocellular carcinoma, miR-18a in gastric cancer, miR-497 in ovarian cancer, miR-1246 in colorectal cancer cells, and miR-21 in prostate cancer through different molecular pathways $(24,33,42-44)$. As one of the bestevaluated miRNA, miR-21 has been reported as an oncogene (45). Knockdown of miR-21 can inhibit angiogenesis in VEGFR2-luc mouse breast tumor model and reverse EMT by targeting PTEN in breast cancer cells $(46,47)$. The significant decrease of miR-21 expression was confirmed by microRNA array and quantitative PCR method in MAD-MB-231-prpM cells. Our data showed that the upregulated expression of miR-21 could partially reverse the inhibition of angiogenesis through the ERK1/2 pathway activation in MAD-MB-231prpM cells, MTDH knockdown inhibited angiogenesis via decreasing the expression level of miR-21.

To further validate the relation of MTDH and angiogenesis in breast cancer samples, we evaluated the angiogenic markers CD31 by using immunohistochemistry. The result showed that MTDH was related with CD31 and the CD31 decreased in samples with the lower expression of MTDH, thus, demonstrating that low expression of MTDH suppressed angiogenesis in breast cancer.

Anti-angiogenesis is considered to be a prospective novel therapeutic strategy for malignant tumors. However, to find an efficient target gene or molecule is still an unsolved problem. As an important oncogene, MTDH plays an important role in tumor progression and prognosis. In this study, we provided evidence that the knockdown of MTDH significantly inhibited angiogenesis. Our findings demonstrate that MTDH is a potential therapeutic target for anti-angiogenesis in breast cancer.

\section{Acknowledgements}

This study was supported by National Natural Science Foundation of China (nos. 30772133, 81172529 and 81272903) and Shandong Science and Technology Development Plan (nos. 2012GZC22115 and 2013GRC31801) to Q.Y.

\section{References}

1. Fan L, Strasser-Weippl K, Li J-J, St Louis J, Finkelstein DM, Yu KD, Chen WQ, Shao ZM and Goss PE: Breast cancer in China. Lancet Oncol 15: e279-e289, 2014.

2. DeSantis C, Ma J, Bryan L and Jemal A: Breast cancer statistics, 2013. CA Cancer J Clin 64: 52-62, 2014.

3. Ellis LM and Fidler IJ: Angiogenesis and Metastasis. Eur J Cancer 32A: 2451-2460, 1996.

4. Bikfalvi A: Significance of angiogenesis in tumour progression and metastasis. Eur J Cancer 31A: 1101-1104, 1995.

5. Fox SB: Tumor angiogenesis and prognosis. Histopathology 30: : 294-301, 1997.

6. Weidner NSJ, Welch WR and Folkman J: Tumor angiogenesis and metastasis - correlation in invasive breast carcinoma. $\mathrm{N}$ Engl J Med 324: 8, 1991.

7. Risau W: Mechanisms of angiogenesis. Nature 386: 671-674, 1997.

8. Su ZZ, Kang DC, Chen Y, Pekarskaya O, Chao W, Volsky DJ and Fisher PB: Identification and cloning of human astrocyte genes displaying elevated expression after infection with HIV-1 or exposure to HIV-1 envelope glycoprotein by rapid subtraction hybridization, RaSH. Oncogene 21: 3592-3602, 2002.

9. Wang $\mathrm{C}$ and Yang Q: Astrocyte elevated gene-1 and breast cancer (Review). Oncol Lett 2: 399-405, 2011.
10. Li X, Kong X, Huo Q, Guo H, Yan S, Yuan C, Moran MS, Shao C and Yang Q: Metadherin enhances the invasiveness of breast cancer cells by inducing epithelial to mesenchymal transition. Cancer Sci 102: 1151-1157, 2011

11. Zhao Y, Kong X, Li X, Yan S, Yuan C, Hu W and Yang Q: Metadherin mediates lipopolysaccharide-induced migration and invasion of breast cancer cells. PLoS One 6: e29363, 2011.

12. Xu C, Kong X, Wang H, et al: MTDH mediates estrogen-independent growth and tamoxifen resistance by down-regulating PTEN in MCF-7 breast cancer cells. Cell Physiol Biochem 33: 1557-1567, 2014

13. Zhang N, Wang X, Huo Q, Li X, Wang H, Schneider P, Hu G and Yang Q: The oncogene metadherin modulates the apoptotic pathway based on the tumor necrosis factor superfamily member TRAIL (tumor necrosis factor-related apoptosis-inducing ligand) in breast cancer. J Biol Chem 288: 9396-9407, 2013.

14. Li J, Zhang N, Song LB, et al: Astrocyte elevated gene-1 is a novel prognostic marker for breast cancer progression and overall patient survival. Clin Cancer Res 14: 3319-3326, 2008.

15. Yoo BK, Emdad L, Su ZZ, Villanueva A, Chiang DY, Mukhopadhyay ND, Mills AS, Waxman S, Fisher RA, Llovet JM, et al: Astrocyte elevated gene-1 regulates hepatocellular carcinoma development and progression. J Clin Invest 119: 465-477, 2009.

16. Liu Y, Su Z, Li G, et al: Increased expression of metadherin protein predicts worse disease-free and overall survival in laryngeal squamous cell carcinoma. Int J Cancer 133: 671-679, 2013.

17. Zhou J, Li J, Wang Z, Yin C and Zhang W: Metadherin is a novel prognostic marker for bladder cancer progression and overall patient survival. Asia Pac J Clin Oncol 8: e42-e48, 2012.

18. Song L, Li W, Zhang H, Liao W, Dai T, Yu C, Ding X, Zhang L and Li J: Over-expression of AEG-1 significantly associates with tumour aggressiveness and poor prognosis in human non-small cell lung cancer. J Pathol 219: 317-326, 2009.

19. Tokunaga E, Nakashima Y, Yamashita N, Hisamatsu Y, Okada S, Akiyoshi S, Aishima S, Kitao H, Morita M and Maehara Y: Overexpression of metadherin/MTDH is associated with an aggressive phenotype and a poor prognosis in invasive breast cancer. Breast Cancer 21: 341-349, 2014.

20. Kong X, Moran MS, Zhao Y and Yang Q: Inhibition of metadherin sensitizes breast cancer cells to AZD6244. Cancer Biol Ther 13: 43-49, 2012.

21. Kikuno N, Shiina H, Urakami S, Kawamoto K, Hirata H, Tanaka Y, Place RF, Pookot D, Majid S, Igawa M, et al: Knockdown of astrocyte-elevated gene-1 inhibits prostate cancer progression through upregulation of FOXO3a activity. Oncogene 26: 7647-7655, 2007.

22. Meng X, Brachova P, Yang S, Xiong Z, Zhang Y, Thiel KW and Leslie KK: Knockdown of MTDH sensitizes endometrial cancer cells to cell death induction by death receptor ligand TRAIL and HDAC inhibitor LBH589 co-treatment. PLoS One 6: e20920, 2011.

23. Hu G, Chong RA, Yang Q, Wei Y, Blanco MA, Li F, Reiss M, $\mathrm{Au}$ JL, Haffty BG and Kang Y: MTDH activation by $8 \mathrm{q} 22$ genomic gain promotes chemoresistance and metastasis of poorprognosis breast cancer. Cancer Cell 15: 9-20, 2009.

24. Fang JH, Zhou HC, Zeng C, Yang J, Liu Y, Huang X, Zhang JP, Guan XY and Zhuang SM: MicroRNA-29b suppresses tumor angiogenesis, invasion, and metastasis by regulating matrix metalloproteinase 2 expression. Hepatology 54: 1729-1740, 2011.

25. Wang X, Zhang N, Huo Q and Yang Q: Anti-angiogenic and antitumor activities of Huaier aqueous extract. Oncol Rep 28: $1167-1175,2012$

26. Su P, Zhang Q and Yang Q: Immunohistochemical analysis of Metadherin in proliferative and cancerous breast tissue. Diagn Pathol 5: 38, 2010.

27. Zhu J, Li X, Kong X, Moran MS, Su P, Haffty BG and Yang Q: Testin is a tumor suppressor and prognostic marker in breast cancer. Cancer Sci 103: 2092-2101, 2012.

28. Wang H, Ach RA and Curry B: Direct and sensitive miRNA profiling from low-input total RNA. RNA 13: 151-159, 2007.

29. Du J, Xu R, Hu Z, Tian Y and Y Z: PI3K and ERK-induced Racl activation mediates hypoxia-induced HIF-1 $\alpha$ expression in MCF-7 breast cancer cells. PloS One 6: e25213, 2011.

30. Liu L, Cao Y, Chen C, Zhang X, McNabola A, Wilkie D, Wilhelm S, Lynch M and Carter C: Sorafenib blocks the RAF/ MEK/ERK pathway, inhibits tumor angiogenesis, and induces tumor cell apoptosis in hepatocellular carcinoma model PLC/ PRF/5. Cancer Res 66: 11851-11858, 2006.

31. Shan B, Li W, Yang S-Y and Li Z-R: Estrogen up-regulates MMP2/9 expression in endometrial epithelial cell via VEGFERK1/2 pathway. Asian Pac J Trop Med 6: 826-830, 2013. 
32. Meng F, Henson R, Wehbe-Janek H, Ghoshal K, Jacob ST and Patel T: MicroRNA-21 regulates expression of the PTEN tumor suppressor gene in human hepatocellular cancer. Gastroenterology 133: 647-658, 2007.

33. Liu LZ, Li C, Chen Q, Jing Y, Carpenter R, Jiang Y, Kung HF Lai $\mathrm{L}$ and Jiang BH: MiR-21 induced angiogenesis through AKT and ERK activation and HIF-1 $\alpha$ expression. PLoS One 6: e19139, 2011.

34. Shapira I, Lee A, Vora R and Budman DR: P53 mutations in triple negative breast cancer upregulate endosomal recycling of epidermal growth factor receptor (EGFR) increasing its oncogenic potency. Crit Rev Oncol Hematol 88: 284-292, 2013.

35. Ma J, Xue Y, Cui W, Li Y, Zhao Q, Ye W, Zheng J, Cheng Y, Ma Y, Li S, et al: Ras homolog gene family, member A promotes p53 degradation and vascular endothelial growth factor-dependent angiogenesis through an interaction with murine double minute 2 under hypoxic conditions. Cancer 118: 4105-4116, 2012.

36. Li X, Kong X, Wang Y and Yang Q: 53BP1 is a novel regulator of angiogenesis in breast cancer. Cancer Sci 104: 1420-1426, 2013.

37. Li C, Li R, Song H, Wang D, Feng T, Yu X, Zhao Y, Liu J, Yu X, Wang Y, et al: Significance of AEG-1 expression in correlation with VEGF, microvessel density and clinicopathological characteristics in triple-negative breast cancer. J Surg Oncol 103 : 184-192, 2011.

38. Bartholomeusz C, Gonzalez-Angulo AM, Liu P, Hayashi N, Lluch A, Ferrer-Lozano J and Hortobágyi GN: High ERK protein expression levels correlate with shorter survival in triple-negative breast cancer patients. Oncologist 17: 766-774, 2012.
39. Chetram MA and Hinton CV: PTEN regulation of ERK1/2 signaling in cancer. J Recept Signal Transduct Res 32: 190-195, 2012.

40. Bartel DP: MicroRNAs: Genomics, biogenesis, mechanism, and function. Cell 116: 281-297, 2004.

41. Anand S and Cheresh DA: Emerging role of micro-RNAs in the regulation of angiogenesis. Genes Cancer 2: 1134-1138, 2011.

42. Yamada N, Tsujimura N, Kumazaki M, Shinohara $H$, Taniguchi K, Nakagawa Y, Naoe T and Akao Y: Colorectal cancer cell-derived microvesicles containing microRNA-1246 promote angiogenesis by activating Smad 1/5/8 signaling elicited by PML down-regulation in endothelial cells. Biochim Biophys Acta 1839: 1256-1272, 2014.

43. Wang W, Ren F, Wu Q, Jiang D, Li H and Shi H: MicroRNA-497 suppresses angiogenesis by targeting vascular endothelial growth factor A through the PI3K/AKT and MAPK/ERK pathways in ovarian cancer. Oncol Rep 32: 2127-2133, 2014.

44. Zheng Y, Li S and Ding Y: The role of miR-18a in gastric cancer angiogenesis. Hepatogastroenterology 60: 5, 2013.

45. Si ML, Zhu S, Wu H, Lu Z, Wu F and Mo YY: miR-21-mediated tumor growth. Oncogene 26: 2799-2803, 2007.

46. Han M, Liu M and Wang Y: Antagonism of miR-21 reverses epithelial-mesenchymal transition and cancer stem cell phenotype through AKT/ERK1/2 inactivation by targeting PTEN. PLoS One 7: 11, 2012.

47. Zhao D, Tu Y, Wan L, Bu L, Huang T, Sun X, Wang K and Shen B: In vivo monitoring of angiogenesis inhibition via down-regulation of mir-21 in a VEGFR2-luc murine breast cancer model using bioluminescent imaging. PLoS One 8: e71472, 2013. 